\title{
Biologia da fase parasitária e de vida livre de adultos de Amblyomma cajennense (Fabricius, 1787) (Acari: Ixodidae) em cães (Canis familiaris L.) sob condições experimentais
}

\author{
Biology of the parasitic and free living phases of Amblyomma \\ cajennense (Fabricius, 1787) (Acari: Ixodidae) adults on dogs (Canis \\ familiaris L.) under experimentaly conditions
}

\author{
Ana Cristina Barros Cardoso, ${ }^{\star}$ Erik Daemon, ${ }^{\star \star}$ Sabrina Sylvain Ribeiro, ${ }^{\star \star \star}$ João Luiz Horácio Faccini ${ }^{\star \star \star \star}$
}

\begin{abstract}
Resumo
Com o objetivo de ampliar as informações sobre a relação parasito-hospedeiro e avaliar o desenvolvimento das fases parasitária e não-parasitária de adultos de Amblyomma cajennense alimentados em cães, foram realizadas infestações, em cinco cães mestiços, utilizando-se dez casais por pavilhão auricular. A fase não-parasitária foi estudada sob condições controladas de laboratório com temperatura de $27 \pm 1^{\circ} \mathrm{C}$, umidade relativa do ar superior a $70 \%$ e escotofase. $\mathrm{O}$ período parasitário médio foi de 14,93 $\pm 5,36$ dias. Do total de 100 casais de Amblyomma cajennense utilizados, 46 fêmeas foram recuperadas. O peso médio das fêmeas ingurgitadas foi de $324,98 \pm 207,47 \mathrm{mg}$ e o período médio de pré-postura e postura foram, respectivamente, de $8,71 \pm 2,40$ e 16,79 $\pm 6,84$ dias. O peso médio da massa de ovos foi de $110,29 \pm 100,79 \mathrm{mg}$ e os índices de eficiência reprodutiva e nutricional foram de $33,67 \%$ e $52,22 \%$, respectivamente. O período de incubação médio foi de $36,79 \pm 6,13$ dias. O percentual de eclosão oscilou em torno de $40 \%$ e o periodo médio de eclosão foi de $14,36 \pm 9,24$ dias.
\end{abstract}

Palavras-chave: Amblyomma cajennense; biologia; cães; infestação experimental.

\begin{abstract}
The development of the parasitic and non parasitic phases of Amblyomma cajennense females on dogs were evaluated. Infestations were carried out on five cross-bred dogs using ten couples of ticks on each ear. The non parasitic phase was studied under controlled conditions $\left(27 \pm 1^{\circ} \mathrm{C}\right.$, relative humidity above $70 \%$ and scotophase). The parasitic period was $14,93 \pm$ 5,36 days. Forty-six engorged females were recovered of a total of 100 unenengorged ones used in the trials only 24 started oviposition. The mean weight of the engorged females was $324,98 \pm 207,47 \mathrm{mg}$ and the preoviposition and oviposition periods were, respectively, $8,71 \pm 2,40$ and $16,79 \pm 6,84$ days. The mean oviposition weight was $110,29 \pm 100,79$ mg and the reproductive and nutricional efficiency indexes were 33,67 and $52,22 \%$, respectively. The incubation period was $36,79 \pm 6,13$ days. The percentage of eclosion was about $40 \%$ and the eclosion period was $14,36 \pm 9,24$ days.
\end{abstract}

Keywords: Amblyomma cajennense; biology; dogs; infestation experimental.

\section{Introdução}

Amblyomma cajennense (Fabricius, 1787) (Acari: Ixodidae), vulgarmente conhecido como carrapato-estrela, é um ixodídeo de regiōes de clima tropical. Em áreas de pastagem da Universidade Federal Rural do Rio de Janeiro (Seropédica, RJ) todos os estágios parasitários são encontrados nos 12 meses do ano, sendo as formas adultas mais freqüentes entre os meses de setembro e dezembro (Serra Freire, 1982). Segundo Aragão (1936), esta espécie possui pouca especificidade. Diversos mamíferos domésticos e silvestres podem ser parasitados, sendo os eqüinos os hospedeiros de preferência.

A importância deste carrapato não se restringe somente ao fato de causar danos diretos ao hospedeiro, como espoliação e irritação, mas também por ser um carrapato de três hospedeiros, o que facilita a eventual transmissão de uma série de agentes patogênicos, entre eles protozoários, vírus e bactérias. Dentro deste contexto, $A$. cajennense atua como vetor de Rickettsia rickettsii, causadora da febre maculosa (Monteiro e Tiriba, 1974 e Lemos et al.,1997). Estes ixodídeos são capa-

\footnotetext{
* Médica Veterinária, Mestranda do Curso de Pós-Graduação em Medicina Veterinária - Parasitologia Veterinária, Instituto de Biologia, Universidade Federal Rural do Rio de Janeiro, BR 465, Km 7, CEP 23890-000, Seropédica, RJ. E-mail: cardosoacb@ufrrj.br.

** Professor Adjunto IV, Departamento de Parasitologia Animal, UFRRJ.

*** Aluna do Curso de Graduação em Medicina Veterinária, UFRRJ. Bolsista de Iniciação Científica - CNPq.

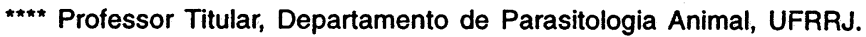


zes de permanecer meses e anos à espera do hospedeiro ideal, o que os tornam importantes na cadeia epidemiológica desta ricketsiose na natureza, garantindo foco endêmico prolongado (Labruna e Pereira, 1998).

Os cães, como animais de estimação, podem ter sua importância na disseminação da febre maculosa e de outras moléstias que podem ser fatais, ao transportarem carrapatos previamente infectados, que neles se alimentam, para o interior de residências humanas.

$\mathrm{Na}$ literatura não há registros relativos a estudos acerca das fases parasitária e não-parasitária de $A$. cajennense utilizando-se cães como hospedeiros. No que se refere a outras espécies de hospedeiros, Rohr (1909) foi o primeiro a fornecer detalhes sobre os principais aspectos da biologia deste ixodideo no Brasil. Smith (1975) investigou alguns parâmetros do ciclo de vida da fêmea de $A$. cajennense alimentada em ovinos. Drummond e Whetstone (1975), utilizando bovinos como hospedeiro, Sanavria et al. (1996) e Prata e Daemon (1997), a partir de infestações experimentais em eqüinos, empreenderam estudos sobre o aspecto da oviposição da referida espécie em condições de laboratório. Olivieri \& Serra Freire $(1984 a, b)$ e Prata et al. $(1996 ; 1997)$ verificaram parâmetros biológicos referentes às fases larval e ninfal, enquanto Daemon e Ishizuca (1992; 1995) analisaram o efeito de diferentes temperaturas sobre a ecdise larval e ninfal de $A$. cajennense mantidas em coelhos.

Este experimento teve por objetivo ampliar as informações sobre a relação parasito-hospedeiro entre $A$. cajennense e o cão doméstico, avaliando o desenvolvimento das fases parasitária e não-parasitária de adultos desde ixodideos alimentados em cães.

\section{Material e métodos}

O presente trabalho foi desenvolvido no Laboratório de Ixodologia do Departamento de Parasitologia Animal na Estação para Pesquisas Parasitológicas W.O. Neitz, da Universidade Federal Rural do Rio de Janeiro, município de Seropédica, estado do Rio de Janeiro, no período de julho de 1998 a fevereiro de 1999.

Foram utilizados cinco cães, adultos, machos, sem raça e idade definidas, que receberam alimentação à base de ração balanceada, água, vermifugação e vacinação adequadas.

A partir de colônias de $A$. cajennense mantidas no Laboratório de Ixodologia foram obtidos 100 casais, através de infestações experimentais em coelhos, segundo a técnica de Neitz et al. (1971). Para a realização da fase parasitária em cães, as infestações foram feitas utilizando-se a técnica anteriormente citada adaptada para a espécie canina. O colar de funil de Holmes (McCunn, 1953), ajustado para caninos, foi empregado com a finalidade de evitar a retirada do saco de pano.

Foram utilizados dez casais de carrapatos com 30 a 40 dias pós-ecdise por pavilhão auricular. Após a infestação foram realizadas aberturas diárias dos sacos de pano para observação do processo de fixação e ingurgitamento dos carrapatos, recolhendo-se as fêmeas ingurgitadas naturalmente desprendidas. Após a coleta, as fêmeas foram levadas ao laboratório, onde foram limpas com pincel de cerdas macias, pesadas em balança analítica e acondicionadas individualmente em placas de Petri, devidamente identificadas, e mantidas em estufa biológica, regulada à temperatura de $27 \pm 1^{\circ} \mathrm{C}$ umidade relativa superior a $80 \%$ e escotofase. A massa de ovos de cada fêmea em postura foi pesada diariamente e subdividida em aliquotas de $100 \mathrm{mg}$, acondicionadas em seringas plásticas descartáveis, devidamente preparadas, vedadas com bucha de algodão e mantidas nas mesmas condições que as fêmeas.

Foram verificados os seguintes parâmetros biológicos: periodo parasitário, percentual de recuperação de fêmeas ingurgitadas, peso médio das fêmeas ingurgitadas, períodos de pré-postura e postura, peso da massa de ovos, peso da quenógina, período de incubação de ovos, período e percentual de eclosão e sobrevivência das larvas. Foram calculados ainda os índices de Eficiência Reprodutiva (IER) e Nutricional (IEN), segundo preconizado por Bennett (1974). Para análise estatística dos dados foi realizada Análise de Variância seguido de Teste de Kruskall-Wallis com nível de $5 \%$ de significância.

\section{Resultados e discussão}

Devido a inexistência de dados relacionados ao ciclo biológico de $A$. cajennense em cães, os valores obtidos foram comparados àqueles provenientes de infestações em eqüinos, considerados hospedeiros primários desta espécie.

Das 100 fêmeas utilizadas para a infestação foram obtidos $46(46 \%)$ exemplares ingurgitados. Observou-se um intenso prurido causado pela ação dos carrapatos, fato que levava os cães a sacudirem a cabeça, provocando esmagamento de fêmeas em adiantada fase de ingurgitamento, mesmo com a utilização do colar funil de Holmes. Sanavria et al. (1996), ao realizarem infestações experimentais com adultos de $A$. cajennense em eqüinos, verificaram um percentual de recuperação igual a $43,57 \%$, valor próximo ao encontrado neste experimento e o mesmo quadro clínico de intenso prurido.

O tempo de ingurgitamento das fêmeas variou de nove a 37 dias $(14,93 \pm 5,36)$ notando-se que mais de $80 \%$ das mesmas foram recuperadas até o 18 dia após a infestação (Figura 1). Smith (1975) relatou período de 12 a 14 dias para ingurgitamento de fêmeas de $A$. cajennense obtidos de diferentes hospedeiros, entre eles eqüinos, sem no entanto especificar o tempo de ingurgitamento em eqüinos. Sanavria et al. (1996) observaram período alimentar de oito a dez dias em uma amostra de 366 fêmeas ingurgitadas. Isto leva a concluir que, quando alimentadas em cães, as fêmeas necessitam de um tempo maior para atingirem o pleno ingurgitamento.

$\mathrm{Na}$ Tabela 1 estão apresentados os resultados referentes ao peso das fêmeas ingurgitadas, os períodos de pré-postura e postura, peso da massa de ovos, IER, IEN e peso da quenógina, destacando-se que, das 46 fêmeas ingurgitadas apenas 24 iniciaram postura $(52,17 \%)$.

Sanavria et al. (1996) e Prata e Daemon (1997) evidenciaram peso médio das fêmeas ingurgitadas de $570,38 \pm 121,43 \mathrm{mg}$ $(360,00-820,00)$ e $816,28 \pm 152,76 \mathrm{mg}(648,00-1077,00)$, respectivamente, todos superiores aos valores encontrados neste experimento, peso médio de $324,98 \pm 207,47 \mathrm{mg}(20,90-$ $700,40)$, mostrando que cães não são plenamente adequados ao desenvolvimento deste ixodídeo quando comparados a hospedeiros naturais. 
Tabela 1: Parâmetros obtidos pelas variáveis referentes à fase não-parasitária de fêmeas de Amblyomma cajennense, sob condições controladas $\left(27 \pm 1^{\circ} \mathrm{C} ; 80 \pm 10 \%\right.$ de umidade, escotofase) alimentadas em cães.

\begin{tabular}{lcccc}
\hline Variáveis & $N^{\circ}$ & \multicolumn{2}{c}{ Limite de variação } & Média \pm desvio- \\
\cline { 3 - 4 } & & Mínimo & Máximo & padrão \\
\hline Peso da fêmea ingurgitada (mg) & 46 & 20,90 & 700,40 & $324,98 \pm 207,47$ \\
Periodo de pré-postura (dias) & 24 & 5 & 15 & $8,71 \pm 2,40$ \\
Periodo de postura (dias) & 24 & 5 & 32 & $16,79 \pm 6,84$ \\
Peso da massa de ovos (mg) & 24 & 1,70 & 317,30 & $110,29 \pm 100,79$ \\
IER (\%) & 24 & 1,60 & 54,92 & $33,67 \pm 16,73$ \\
IEN (\%) & 24 & 8,13 & 80,73 & $52,22 \pm 21,09$ \\
Peso da quenógina (mg) & 24 & 18,60 & 381,60 & $107,53 \pm 87,70$ \\
\hline
\end{tabular}

IER - Índice de Eficiência Reprodutiva.

IEN - Índice de Eficiência Nutricional.

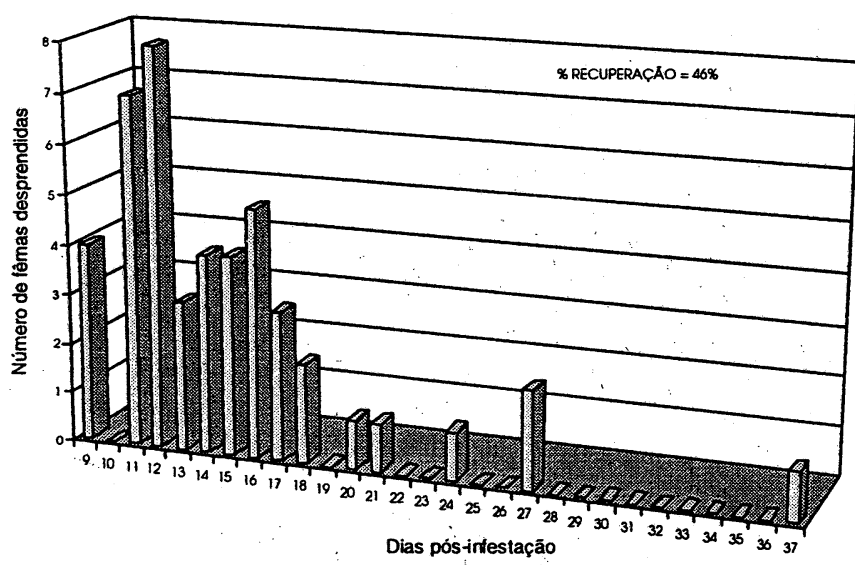

Figura 1: Ritmo de queda de fêmeas ingurgitadas de Amblyomma cajennense a partir de infestação experimental em cães.

O periodo de pré-postura observado por Travassos e VallejoFreire (1944), quatro a 12 dias, com média de 7,3 dias e Sanavria et al. (1996), cinco a 11 dias $(7,07 \pm 1,24$ dias) foram bem próximos ao encontrado neste experimento, demonstrando pouca influência do hospedeiro sobre esta variável.

O período médio de postura evidenciado por Travassos e Vallejo-Freire (1944), de 23,3 (10-32 dias), mostrou-se superior ao obtido neste experimento.

No que concerne ao ritmo de postura, ressalta-se que a maioria dos ovos foram postos até o $19^{\circ}$ dia $(80,37 \%)$ (Figura 2$)$. Prata e Daemon (1997) observaram que $50 \%$ dos ovos já haviam sido postos até o $5^{\circ}$ dia de postura, enquanto que neste experimento apenas $15,05 \%$ dos ovos foram postos neste mesmo período, e a média de $50 \%$ só foi alcançada no $13^{\circ}$ dia.

Sanavria et al. (1996) e Prata e Daemon (1997) observaram, infestando experimentalmente eqüinos, um peso médio da massa de ovos de $236,40 \pm 53,20 \mathrm{mg}(142,27-362,50 \mathrm{mg}) \mathrm{e}$ $388,77 \pm 94,37 \mathrm{mg}(199,20-501,90)$, respectivamente, valores acentuadamente superiores aos do presente trabalho. Do mesmo modo, os Índices de Eficiência Reprodutiva e Nutricional, quando comparados com os valores encontrados por Sanavria et al. (1996) e Prata e Daemon (1997), mostraram-se baixos, caracterizando a baixa eficiência biológica de fêmeas de $A$. cajennense alimentadas em cães.

Os resultados referentes aos períodos de incubação e eclosão e os percentuais de eclosão e longevidade de larvas podem ser vistos na Tabela 2. Ressalta-se que do total de fêmeas ingurgitadas coletadas dos cães, somente 24 $(52,17 \%)$ iniciaram postura, totalizando $24 \%$ da infestação inicial, e que apenas os ovos provenientes de 14 fêmeas $(58,33 \%)$ resultaram em eclosão larval. Considerando que foram utilizadas 100 fêmeas nas infestações iniciais, somente $14 \%$ tiveram sucesso na produção de larvas. O período de incubação $(36,79 \pm 6,13$ dias) apresentou-se semeIhante aos valores observados por Sanavria et al. (1996), mas quando comparados com o percentual de eclosão encontrados por estes mesmos autores $(69,99 \pm 12,20)$, foram inferiores $(41,66 \pm 30,15)$.

Tabela 2: Parâmetros obtidos pelas variáveis referentes ao desenvolvimento de ovos de fêmea de Amblyomma cajennense, sob condiçōes controladas $\left(27 \pm 1^{\circ} \mathrm{C} ; 80 \pm 10 \%\right.$ de umidade, escotofase) alimentadas em cães.

\begin{tabular}{lcccc}
\hline Variáveis & $N^{2}$ & \multicolumn{2}{c}{ Limite de variação } & Média \pm desvio- \\
\cline { 3 - 4 } & & Mínimo & Máximo & padrão \\
\hline Periodo de incubação (dias) & 14 & 28 & 48 & $36,79 \pm 6,13$ \\
Periodo de eclosão (dias) & 14 & 1 & 35 & $14,36 \pm 9,24$ \\
Percentual de edosão (\%) & 14 & 0,86 & 90 & $41,66 \pm 30,15$ \\
Mortalidade (dias) & 13 & 32 & 173 & $92,23 \pm 46,93$ \\
\hline
\end{tabular}

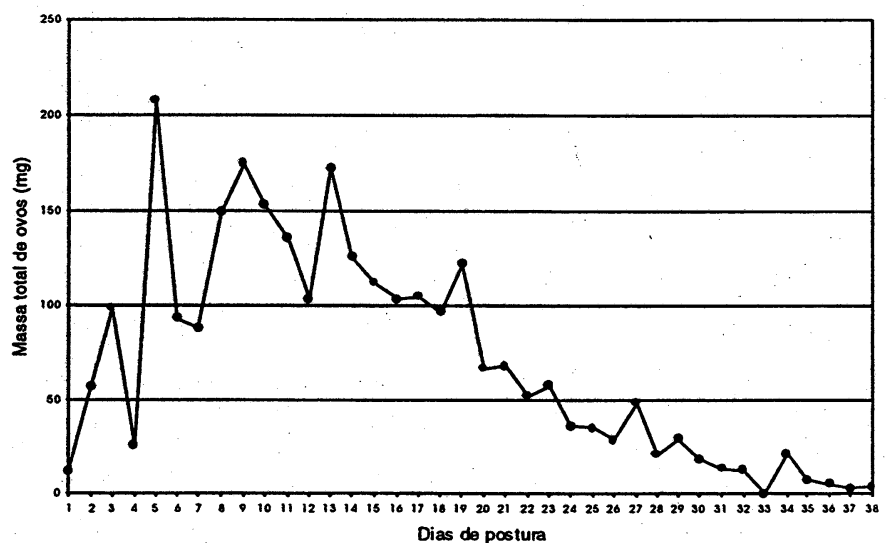

Figura 2: Ritmo de postura de Amblyomma cajennense a partir de infestação experimental em cães.

Os resultados obtidos neste experimento permitem inferir que o desenvolvimento de fêmeas de $A$. cajennense, alimentadas em cães, fica prejudicado quando comparados com os observados em eqüinos, considerados hospedeiros prediletos. No entanto, mantém o cão na lista de hospedeiros alternativos secundários, não eliminando a possibilidade deste ser um elo entre o homem e diversos animais silvestres considerados reservatórios de agentes patogênicos. 


\section{Agradecimentos}

Conselho Nacional de Desenvolvimento Científico e Tecnológico (CNPq); Cargil S/A, pelo fornecimento de ração para os animais; Centro de Controle de Zoonoses da Prefeitura do Rio de Janeiro (CCZ).

\section{Referências bibliográficas}

ARAGÃO, H.B. Ixodidas brasileiros e de alguns paizes limitrophes. Mem. Inst. Oswaldo Cruz, v. 31, n. 4, p. 759-845, 1936.

BENNETT, F.G. Ovoposition of Boophilus microplus (Canestrini) (Acarida: Ixodidae). I. Influence of tick size on egg production. Acarologia, v. 16, n. 1, p. 52-61, 1974.

DAEMON, E., ISHIZUKA, A.C. Efeito de diferentes temperaturas sobre a ecdise lanval de Amblyomma cajennense (Fabricius, 1787) (Acarina: Ixodidae). Rev. Bras. Parasitol. Vet., v. 1, p. 105-107, 1992.

Efeito de diferentes temperaturas sobre a ecdise ninfal de Amblhyoma cajennense (Acarina: Ixodidae). Rev. Bras. Ciênc. Vet., v. 2, n. 1, p. 7-9, 1995.

DRUMMOND, R.O., WHETSTONE, T.M. Oviposition of the cayenne tick, Amblyomma cajennense(F.), in the laboratory. Ann. Entomol. Soc. Amer., v. 68, n. 2, p. 214-216, 1975.

LABRUNA, M.B., PEREIRA, M.C. Febre maculosa: aspectos clínicoepidemiológicos. Rev. Clin. Vet., n. 12, p. 19-23, 1998.

LEMOS, E.R.S., MACHADO, R.D., PIRES, F.D.A., MACHADO, S.L., COSTA, L.M.C., COURA,J.R. Rickettsiae-infected in an endemic area of spotted fever in the state of Minas Gerais, Brazil. Mem. Inst. Oswaldo Cruz, v. 92, n. 4, p. 477-481, 1997.

McCUNN, J. Hobday's Surgical Diseases of Dog and Cat. 6. ed. London: Baillière Tinndall and Cox, 1953, 453p.

MONTEIRO, E.V.L., TIRIBA, A.C. Doenças infecciosas e parasitárias. 6. ed., Guanabara Koogan, p. 236-249, 1974.

NEITZ, W.O., BOUGHTON, F., WALTERS, H.S. Laboratory investigations on the life cycle of the karoo paralysis tick (Ixodes rubicundus Neumann, 1904). Onderst. J. Vet. Res., v. 38, n. 3, p. 215-224, 1971.
OLIVIERI, J.A., SERRA FREIRE, N.M. Estádio larval do ciclo biológico de Amblyomma cajennense. Arq. Univ. Fed. Rio de J., v. 7, p. 139147, 1984a.

Estádio lanval do ciclo biológico de Amblyomma cajennense. Arq. Univ. Fed. Rio de J., v. 7, p. 139-147, 1984 b.

PRATA, M.C.A., ALONSO, L.S., SANAVRIA, A. Parâmetros biológicos do estágio ninfal de Amblyomma cajennense (Fabricius, 1787) (Acari: Ixodidae) em coelhos. Rev. Bras. Ciênc. Vet, v. 3, n. 2, p. 55-57, 1996.

- Parâmetros biológicos do estágio larval de Amblyomma cajennense (Fabricius, 1787) (Acari: Ixodidae) em coelhos. Rev. Bras. Ciênc. Vet., v. 4, n. 1, p. 5-8, 1997.

PRATA, M.C.A., DAEMON, E. Determinação do número de ovos por grama de postura de Amblyomma cajennense (Fabricius, 1787) (Acari: Ixodidae). Rev. Bras. Ciênc. Vet., v. 4, n. 2, p. 81-82, 1997.

ROHR, C.J. Estudos sobre Ixodidas no Brasil. Rio de Janeiro: Inst. Osw. Cruz, 1909, 220 p.

SANAVRIA, A., PRATA, M.C.A., MORAIS, M.C. Determinação de alguns parâmetros biológicos de Amblyomma cajennense (Fabricius, 1787) (Acari: Ixodidae) em infestação artificial de eqüino. Arq. Fac. Vet. UFRGS, v. 24, n. 2, p. 79-86, 1996.

SERRA FREIRE, N.M. Epidemiologia de Amblyomma cajennense. ocorrência estacional e comportamento dos estádios não parasitários em pastagens do Estado do Rio de Janeiro. Arq. Univ. Fed. Rur. Rio de Jan., v. 5, p. 187-193, 1982.

SMITH, M.W. Some aspects of the ecology and life cycle of Amblyomma cajennense (Fabricius, 1787) in Trinidad and their influence on tick control measures. Ann. Trop. Med. Parasitol., v. 69, n. 1, p. 121129, 1975.

TRAVASSOS, J., VALLEJO-FREIRE, A. Criação artificial de $A$. cajennense para preparo de vacina contra a febre maculosa. Mem. Inst. Butantan, v. 18, p. 145-235, 1944. 